NBER WORKING PAPER SERIES

\title{
A THEORY OF VOLUNTARY TESTING AND SELF-ISOLATION IN AN ONGOING
} PANDEMIC

Thomas F. Hellmann

Veikko Thiele

Working Paper 27941

http://www.nber.org/papers/w27941

\author{
NATIONAL BUREAU OF ECONOMIC RESEARCH \\ 1050 Massachusetts Avenue \\ Cambridge, MA 02138 \\ October 2020
}

I thank the Said Business School for its research support. The views expressed herein are those of the authors and do not necessarily reflect the views of the National Bureau of Economic Research.

At least one co-author has disclosed a financial relationship of potential relevance for this research. Further information is available online at http://www.nber.org/papers/w27941.ack

NBER working papers are circulated for discussion and comment purposes. They have not been peer-reviewed or been subject to the review by the NBER Board of Directors that accompanies official NBER publications.

(C) 2020 by Thomas F. Hellmann and Veikko Thiele. All rights reserved. Short sections of text, not to exceed two paragraphs, may be quoted without explicit permission provided that full credit, including $\odot$ notice, is given to the source. 
A Theory of Voluntary Testing and Self-isolation in an Ongoing Pandemic

Thomas F. Hellmann and Veikko Thiele

NBER Working Paper No. 27941

October 2020

JEL No. D8,I12

\begin{abstract}
Thinking beyond Covid-19, there is a growing interest in what economic structures will be needed to face ongoing pandemics. In this paper we focus on the diagnostic problem and examine a new paradigm of voluntary self-testing by private individuals. People without symptoms face daily choices of either taking the risk of going out (to work and socialize), versus staying at home in self-isolation. Our theory shows that two types of people voluntary test themselves: those who otherwise would have self-isolated, and those who would have gone out indiscriminately. Our central insight is that the equilibrium infection risk falls when home-based testing becomes cheaper and easier to use, even if tests are not always accurate. Our results challenge the clinical mainstream view that diagnostic testing is a prerogative of the medical profession, and supports the notion that frequent self-testing is vital for an economy facing an ongoing pandemic.
\end{abstract}

\author{
Thomas F. Hellmann \\ Saïd Business School \\ University of Oxford \\ Park End Street \\ Oxford, OX1 1HP \\ United Kingdom \\ and NBER \\ Thomas.Hellmann@sbs.ox.ac.uk \\ Veikko Thiele \\ School of Business \\ Queen's University \\ Goodes Hall \\ 143 Union Street \\ Kingston, Ontario \\ Canada \\ vthiele@business.queensu.ca
}

A data appendix is available at http://www.nber.org/data-appendix/w27941 


\section{Introduction}

While Covid-19 has generated public awareness about the economic challenges of pandemics, it is only one of the many threats pandemics pose to the economy. Covid-19 was preceded by SARS, Ebola, and AIDS, and is likely to be followed by mutations of the Coronavirus and/or other infectious diseases. Hence an emerging debate about what structures will be need in the presence of on-going pandemics, about what the "new normal" will or should look like? Much attention has been given to the development of new vaccines and drugs. However, it remains unclear how effective they will be, and how broadly they can be deployed. Consequently, there is an increased interest in diagnostic testing. Agrawal et al. (2020), and Gans (2020a) argue that pandemics are essentially an information problem, especially when infections are transmitted by people without symptoms (aka asymptomatic transmission) 11 The major disruptions to economic life occur because people lack information about who is infected, and when. What is the economic role of diagnostic testing in the "new normal" economy?

There is considerable disagreement about the proper approach to testing. At the risk of simplifying, there are two schools of thought that we will call the "clinical mainstream view" versus the "public health view". The mainstream view is that diagnostic tests are a responsibility of the medical system. Testing should be performed by medically trained staff and should occur at the point of care (e.g., hospitals), with the help of approved labs. Diagnostic accuracy is considered extremely important, as it guides all subsequent medical interventions. Two key measures are "sensitivity" which concerns the absence of false negatives (where infected people are erroneously found to be free of the virus) and "specificity" which concerns the absence of false positives (where uninfected people are erroneously found to have the virus). The clinical mainstream view is deeply sceptical about any testing outside of clinical setting. For example, the home page of the Federal Trade Commission provides the following warning ${ }^{2}$ "Be wary of ads for test kits. Most test kits being advertised have not been approved by the FDA and aren't necessarily accurate."

Some public health experts beg to differ. They argue that testing accuracy is not necessarily the most important aspect. Instead what matters is to quickly identify as many infected people as early as possible, before they further spread the disease ${ }^{3}$ Dr. Michael Mina, from the Harvard Chan School of Public Health, argues: $4^{4}$ "As long as you're using the test on a pretty frequent basis, you will be more likely than not to catch the person on the day they might go out and transmit. And they'll know to stay home." Carroll (July 28th, 2020, NYT) further argues: "These tests aren't perfect, but that's not the point

\footnotetext{
${ }^{1}$ The CDC estimated in July 2020 that $40 \%$ of Covid-19 transmissions are asymptomatic; other estimates range from $25 \%$ to $80 \%$ (Plater, 2020).

${ }^{2}$ See https://www.ftc.gov/coronavirus/scams-consumer-advice

${ }^{3}$ For example, as of September 1, 2020, Health Canada is considering approving home tests to screen for Covid-19, even though home tests are expected to be less accurate than laboratory tests; see https://www.cbc.ca/news/politics/ health-canada-covid-home-test-1.5707740.

${ }^{4}$ Quoted by Richard Harris, NPR, July 22nd, 2020; see https://www.npr.org/sections/health-shots/ 2020/07/22/893931848/rapid-cheap-less-accurate-coronavirus-testing-has-a-placescientists-say
} 


\section{[...] every single case we identify is better than not. We can isolate that person from the population and}

prevent infections."

This debate raises some interesting questions about the economic role of testing. Is testing accuracy the most important, or do other aspects such as ease of use and price matter more from an economic perspective? How important is testing accuracy if all people are rational and fully understand the limitations of a test? What if some naïve consumers do not understand these imperfections?

Currently diagnostic testing is undergoing a paradigm shift. Numerous start-ups and established companies are introducing new testing technologies that are faster and cheaper than the current standard approach (called PCR), although they typically are not more accurate (Meyer and Madrigal, 2020; Hellmann, 2020). Most important, testing is no longer just a medical tool for diagnosis, it is also becoming an economic decision tool. There is a new demand for testing that is administered outside of clinical settings, including self-testing at home. Unlike clinical tests which are given when patients have symptoms, and are administered by qualified staff, home-based tests are for asymptomatic private individuals. They self-administer them to make daily decisions about whether to "go out" and participate in public life, or "stay at home" and self-isolate. This simple choice is at the core of the pandemic problem. People who stay at home don't infect others and find out in due time whether they get ill or not. By contrast, people who go out risk getting infected, and risk infecting others. The choice of "going out" should be interpreted broadly, to include going out to work (to generate income), going out to socialize (e.g., visit friends, look after relatives), and going out to consume (e.g., go shopping, eat out).

In this paper we use economic theory to inquire about the private demand for self-testing. As relatively little is known about voluntary self-testing and self-isolation, we ask three sets of questions:

(i) What is the structure of the private demand for testing? What types of people are most interested in regularly buying tests to verify their health status? How does the price and ease of use affect this?

(ii) How important is the accuracy of the test? What are the implications of false negatives and false positives? How do rational people respond testing inaccuracies? What if people are naïve?

(iii) What determines the equilibrium infection risk in an on-going pandemic? How does the availability of home-based tests affect this risk?

This paper develops a formal theoretical model for understanding the new paradigm of voluntary self-testing and self-isolation. We are not interested in short-term dynamics, such as predicting peaks in a pandemic outbreak, or "flattening the curve". Instead we focus on long-term steady state equilibria where people make recurring decisions in the face of ongoing risks. Technically, a steady state means a constant infection rate, also known as " $R=1 "$. Gans (2020b) argues that most behavioral models of pandemics converge to such $R=1$ equilibria. One advantage for focussing on this steady state is that it allows us to derive analytical solutions. Most important, conceptually we are precisely interested in long-run equilibria where pandemics are an on-going challenge, what we call the "new normal". 
Specifically, we consider private individuals making recurring decisions whether to go out into public places, versus stay at home to self-isolate. Going out permits the usual economic activities and gives people positive utility, whereas self-isolation avoids getting infected and infecting others. The formal model uses highly stylized assumptions about the medical illness progression. Importantly, the decision to go out versus self-isolate is made by people who show no symptoms. In the benchmark model they make this decision without testing. In the main model we introduce voluntary self-testing. However, tests can be inaccurate and may generate type I (false positive) and type II (false negative) errors.

We obtain three main sets of results. First, we show that there are two types of people who self-test, those who in the absence of testing would self-isolate, and those who would always go out. The lower the price of a test, and the easier its use, the more people of both types switch to regular self-testing.

Second, we show that tests remain economically valuable even if not perfectly accurate. The mainstream clinical view frequently warns against false negatives, where people go out erroneously thinking they are not infectious. However, the implicit comparison in this argument is a perfect test where no false negatives occur. Our model has a more realistic comparison, where the alternative to an imperfect test is no test at all. Some of the people with false positives would have stayed at home, but others would have gone out anyway. As long as an imperfect test provides some information, it helps to keep higher risk people at home, and allows lower risk people to go out. Rational people who fully understand inaccuracies reduce their demand if tests are less accurate. However, naïve consumers may not understand inaccuracies and put too much trust into the test. A surprising result is that having more naïve people in the economy increases self-testing and reduces the equilibrium infection risk.

Third, self-testing plays an important roles because it affects who goes out and who self-isolates. It changes how contagious people in public places are, and thus what the equilibrium infection risk is. Positive test results convince high-risk people to self-isolate, and negative test results encourage lowrisk people to go out. Importantly, tests do not have to be perfectly accurate to reduce the equilibrium infection risk. Probably the most important result is that lowering the price of testing reduces the equilibrium infection risk, and increases social welfare (as measured by the sum of utilities of all agents in the economy).

While our paper contributes to the rapidly exploding literature on Covid-19, our focus is on understanding on-going pandemics. Our model structure is inspired by SIR/SEIR models, which are the workhorses of mathematical epidemiologists (Brauer and Castillo-Chavez, 2012). Berger et al. (2020) extend the SEIR model to incorporate testing. Eichenbaum et al. (2020) also incorporate testing in a SIR model which they embed in a macro model. In all this literature, testing is introduced as an exogenous factor. Our model differentiates itself from the prior literature by considering the endogenous adoption of testing. We look at individual choices and derive what kind of individuals choose to purchase tests, and under what circumstances. Our model thus captures the emerging paradigm of self-testing by private individuals. Note also that SIR/SEIR models focus on modeling epidemic outbreaks. These models contain rich dynamics but typically do not involve any utility maximization. We use a utility-based choice model which allows us to perform an economic welfare analysis. Our approach of focusing not on med- 
ical but economic choices is in the tradition of Gersovitz and Hammer (2004). We share the inclusion of altruistic preferences with Farboodi et al. (2020). Our interest in the micro foundations of self-isolation build on Toxvaerd (2020) and Rachel (2020). Finally, note that our results are in line with the popular writings of Nobel laureate Paul Romer. He argues that ubiquitous testing should become a central strategy for the Covid-19 crisis, and that people should spend more money on tests than on soda 5

\section{Benchmark Individual Choice Model}

In this section we introduce a simple benchmark model. We examine individuals' choices about voluntary self-isolation and derive the resulting equilibrium infection risk of an on-going pandemic. The benchmark model deliberately omits the possibility of self-testing, which we introduce in Section 4 . All proofs are in the online appendix, which is available on the authors' websites.

Consider a dynamic economy with many individuals, an infinite number of dates $t$, and a discount rate $\delta$ (and let $\bar{\delta} \equiv 1-\delta$ ). Every date people face a choice between either "self-isolating" or "going out". Self-isolation means avoiding contact with other people, such as staying (and possibly working) at home. We assume that anyone self-isolating cannot get infected, nor can they infect others. We normalize the date utility of self-isolation to zero. The date utility of going out is denoted by $y$. This can differ across individuals, so we assume some general distribution $\Omega(y)$.

In our model infections occur without people realizing it (aka asymptomatic transmission). We use the following stylized disease progression. Suppose an individual who is going out gets infected at date $t$. At date $t+1$ this individual remains asymptomatic, i.e., s/he doesn't know if s/he is infected (unless selftesting, see Section 44. If s/he goes out at $t+1$, s/he may infect others. Further exposure to contagious people does not change his/her infection status. At $t+2$, infected people become ill and cannot go out. Being ill is associated with a disutility $z$. We assume that all infected people recover at $t+3$. They are no longer contagious and can go out again. However, we assume no immunity, so that people can get infected again ${ }^{6}$ We denote the infection risk in the economy by $\phi$ (and let $\bar{\phi} \equiv 1-\phi$ ). This is defined as the probability that someone uninfected going out and gets infected. The purpose of the model is to derive the equilibrium properties of $\phi$.

Infecting others is an externality that we allow people to partially internalize. If people were purely selfish, they would never self-test. This is because the test has no medical role. We allow people to have a social conscience and feel remorse if they realize they might have infected others. Specifically, if someone discovers at date $t+2$ that they are ill (which means they got infected at date $t$ ), and they went out at date $t+1$, then their ex-post remorse for going out is characterized by a disutility $x$. Rationally anticipating this at date $t+1$, the expected disutility of going out is $\delta \phi x$. Ex-ante, think of this as people's sense of responsibility.

\footnotetext{
${ }^{5}$ See https://paulromer.net/if-tests-were-sodas/ and Peto et al. (2020).

${ }^{6}$ While these assumptions are not medically accurate, they help to focus on the key trade-offs. The assumptions that no one dies, and that there is no immunity, are necessary to guarantee steady states.
} 
In our benchmark model individuals can choose one of two possible steady-state strategies. The first strategy is to go out whenever possible, we call this the "GO" strategy. Individuals go out even though they know they might be contagious to others. The utility is given by

$$
U^{G O}=\phi\left[y+\delta\left[-x-z+\delta\left(y+\delta U^{G O}\right)\right]\right]+\bar{\phi}\left[y+\delta U^{G O}\right]
$$

which in steady state implies

$$
U^{G O}=\frac{\left(1+\phi \delta^{2}\right) y-\phi \delta(x+z)}{\bar{\delta}+\phi \delta\left(1-\delta^{2}\right)} .
$$

The second strategy is to stay home, we call this the self-isolation ("SI") strategy. The utility is given by $U^{S I}=0.7$

\section{Proposition 1 There exists a critical value $Y_{1}$ such that}

(i) For all $y<Y_{1}$ individuals self-isolate $(S I)$.

(iii) For all $y \geq Y_{1}$ individuals always go out $(G O)$.

The critical value $Y_{1}$ is increasing in $\phi, x$, and $z$.

Individuals with a high utility of going out will always try to do so; individuals with a low utility of going out will self-isolate. Individuals switch from $G O$ to $S I$ (higher $Y_{1}$ ) with a higher chance to get infected (higher $\phi$ ), more remorse infecting someone else (higher $x$ ), or a more severe illness (higher $z$ ).

\section{Benchmark Equilibrium Model}

We now examine what determines the equilibrium infection risk $\phi$ in the benchmark model. This requires the derivation of two equilibrium curves that we call the contagiousness curve (CO-curve) and the infection curve (IN-curve). The CO-curve measures how contagious people are that go out. Specifically, we define $\mu$ as the average contagiousness of an individual going out. We will explain how this depends on the infection risk $\phi$, so that the CO-curve is a function $\mu_{C O}(\phi)$. The IN-curve measures how likely it is for an uninfected individual going out, to become infected. This depends on the contagiousness of the individuals s/he gets exposed to, so that the IN-curve is a function $\phi_{I N}(\mu)$. The equilibrium is the intersection of the CO-curve and the IN-curve - technically the fixed point of $\mu_{C O}(\phi)$ and $\phi_{I N}(\mu)$.

We first derive the CO-curve. We assume that people cannot observe the history of others and get exposed to a set of people that are each contagious with probability $\mu$. In the online appendix we show that in the steady state, a fraction $\varpi^{G O \mid 0}=\frac{1}{1+2 \phi}$ of $G O$ people is not infected, a fraction $\varpi^{G O \mid 1}=\frac{\phi}{1+2 \phi}$ is infected but asymptomatic, and a fraction $\varpi^{I L L}=\frac{\phi}{1+2 \phi}$ is ill and stays home. The CO-curve is therefore given by

$$
\mu_{C O}(\phi)=\frac{\varpi^{G O \mid 1}}{\varpi^{G O \mid 0}+\varpi^{G O \mid 1}}=\frac{\phi}{1+\phi} .
$$

\footnotetext{
${ }^{7}$ In Section 5.3 we consider a additional "partial isolation" strategy.
} 


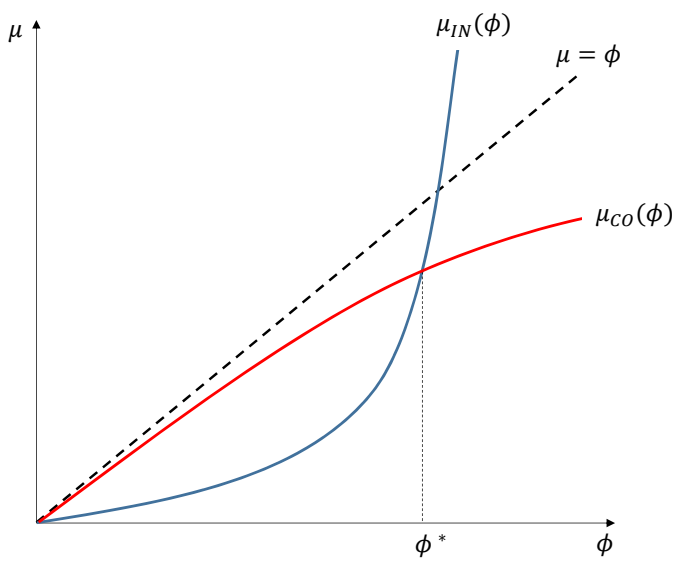

Figure 1: Benchmark Model Equilibrium

The CO-curve is increasing in $\phi$, concave, and satisfies $\mu_{C O}(0)=0$.

To derive the probability of getting infected (i.e., the IN-curve), we use a simple highly stylized infection model. If an individual goes out, s/he meets $M \geq 1$ of other people, each of which may be contagious with probability $\mu . M$ is a measure of the contact level for people going out. There also is an imperfect shielding technology which for any encounter prevents an infection with probability $\lambda$ (e.g. wearing a face masks). The probability of getting infected by any encounter is thus $(1-\lambda) \mu$. For simplicity we treat $M$ and $\lambda$ as exogenous, but show in Section 5.4 how $M$ and $\lambda$ can be endogenized without changing the key insights from our model.

The probability of not getting infected by any encounter on a given day is given by $[1-(1-\lambda) \mu]^{M}$. Thus the probability of getting infected on a given day is

$$
\phi_{I N}(\mu)=1-[1-(1-\lambda) \mu]^{M} .
$$

$\phi_{I N}$ is increasing in $M$ and decreasing in $\lambda$. Moreover, $\phi_{I N}(0)=0$, and $\phi_{I N}(\mu)$ is always increasing in $\mu$, as shown in Figure $1^{8}$ The equilibrium is where the CO- and IN-curves intersect.

Proposition 2 There exists a threshold $\widetilde{M}$ such that for $M<\widetilde{M}$ the only stable equilibrium is $\phi^{*}=0$. For $M>\widetilde{M}$ there exists an equilibrium with $\phi^{*}>0$. A higher $M$ and a lower $\lambda$ shift the IN-curve outward, resulting in a higher equilibrium infection risk $\phi^{*}$.

Proposition 2 shows that if the contact level $M$ is low, then $\phi=0$ is a stable equilibrium. However, for higher levels of $M$, it is no longer stable. Whenever the $\mu_{C O}$ curve lies above the $\mu_{I N}$ curve, a small flare-up in infections quickly raises the average contagiousness of the public pool. In this case the only stable steady state has a positive infection risk $\phi^{*}>0$, i.e., an ongoing pandemic. Henceforth

\footnotetext{
${ }^{8}$ Strictly speaking, Figure 1 shows the inverse function where for each infection risk $\phi_{I N}$ on the horizontal axis, the required contagiousness rate $\mu$ is shown on the vertical axis.
} 


\begin{tabular}{|l|l|l|l|l|}
\hline Person is & Testing says & Decision & Without test would have self-isolated & Without test would have gone out \\
\hline \multirow{2}{*}{ Infected } & Correct positive & Self isolate & Same outcome as without test & Better outcome than without test \\
\cline { 2 - 5 } & False negative & Go out & Worse outcome than without test & Same outcome than without test \\
\hline \multirow{2}{*}{ Not infected } & False positive & Self isolate & Same outcome than without test & Worse outcome than without test \\
\cline { 2 - 5 } & Correct negative & Go out & Better outcome than without test & Same outcome than without test \\
\hline
\end{tabular}

Figure 2: Effect of False and Correct Tests

we focus on this more interesting case where $M>\widetilde{M}$. Higher contact levels (higher $M$ ), and less effective shielding technologies (lower $\lambda$ ), shift the IN-curve outwards, increasing equilibrium infection risk. Intuitively, more contact and less shielding makes going out riskier because of a higher transmission risk.

\section{Voluntary Testing}

We now introduce the possibility that individuals use a test to verify whether they are infected. We are not interested in clinical tests for symptomatic people, but tests that asymptomatic people regularly selfadminister to decide whether to go out or self-isolate. Such tests may be inaccurate, generating both false positives (type I error) and false negatives (type II error). We ask who would purchase such tests, what effect they have on infection rates, and what the importance of type I/II errors are.

In the model tests are only used by asymptomatic people who went out the date before. Let $c$ be the disutility of using a test, which depends on its price and the difficulty of administering it. Let $\alpha$ be the probability of a false positive, and $\beta$ of a false negative test result. There are four possible outcomes:

(i) With $(1-\phi)(1-\alpha)$ : person is uninfected, test correctly negative.

(ii) With $(1-\phi) \alpha$ : person is uninfected, test falsely positive.

(iii) With $\phi(1-\beta)$ : person is infected, test correctly positive.

(iv) With $\phi \beta$ : person is infected, test false negative.

We assume that the test is informative, i.e., $\alpha, \beta \in\left[0, \frac{1}{2}\right)$.Testing is only useful if it generates information that affects decisions. A person who will always go out irrespective of the result would never purchase a test; similar for someone who always self-isolates. We can therefore focus on individuals who go out with a negative but self-isolate with a positive test outcome.

The net effect of an imperfect test should be evaluated against the alternative of no test. For example, people who would otherwise have self-isolated now go out if they get a false negative test result. However, for people who would have gone out anyway, a false negative doesn't actually change their behavior. Figure 2 explains more generally how and when an imperfect test affects behavior. 


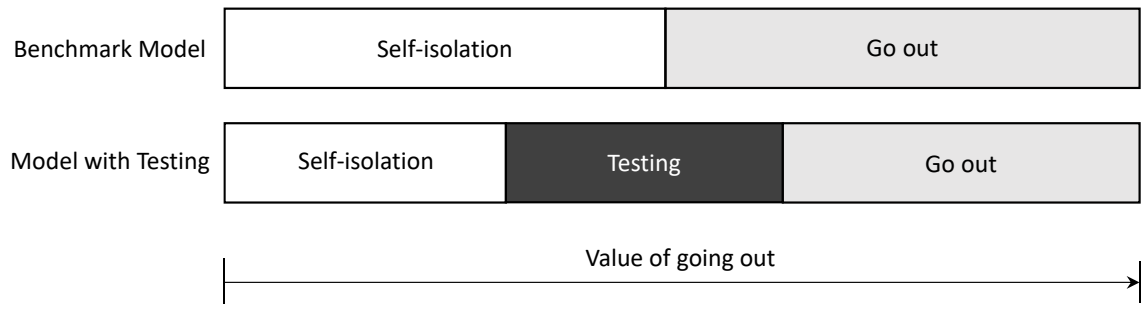

Figure 3: Comparison of Equilibrium Choices

Individuals are fully rational and understand these test limitations (we relax this assumption in Section 5.1). With a testing strategy (called TE), the utility is given by

$$
\begin{aligned}
U^{T E}= & -c+\bar{\phi} \bar{\alpha}\left(y+\delta U^{T E}\right)+\bar{\phi} \alpha \delta\left(y+\delta U^{T E}\right)+\phi \bar{\beta}\left(\delta\left(-z+\delta\left(y+\delta U^{T E}\right)\right)\right) \\
& +\phi \beta\left(y+\delta\left(-x-z+\delta\left(y+\delta U^{T E}\right)\right)\right) .
\end{aligned}
$$

The first term measures the disutility cost of the test. The remaining four terms correspond to the four possibly outcomes described above. In steady state the utility is given by

$$
U^{T E}=\frac{\left[\bar{\phi}(1-\alpha \bar{\delta})+\phi \delta^{2}\right] y-\phi[\beta(\delta x-y)+\delta z]-c}{1-\delta\left[\bar{\phi}(1-\alpha \bar{\delta})+\phi \delta^{2}\right]} .
$$

The utility of testing is decreasing in its costs $c$. Moreover, it is decreasing in $\alpha$ and $\beta$, indicating that less accurate tests are less valuable to rational people.

The next proposition identifies the optimal choices for individuals when self-testing is available. For brevity's sake, we focus on the more interesting case where at least some individuals test. This requires $z<z^{*}$, where $z^{*}$ is characterized in the online appendix.

Proposition 3 There exists a critical testing cost $\widehat{c}$ such that for $c \geq \widehat{c}$ no one does testing. For $c<\widehat{c}$ the optimal choices are as follows:

(i) For all $y<\widehat{Y}_{1}$ individuals self-isolate $(S I)$.

(ii) For all $\widehat{Y}_{1} \leq y<\widehat{Y}_{2}$ individuals test (TE).

(iii) For all $y \geq \widehat{Y}_{2}$ individuals go out $(G O)$.

The critical value $\widehat{Y}_{1}$ is increasing in $c, \alpha$, and $\beta$, and $\widehat{Y}_{2}$ is decreasing in $c$, $\alpha$, and $\beta$.

Figure 3 illustrates the equilibrium choices for the benchmark model, and the model with testing, for different values of $y$. 
The key insight from Proposition 3 is that testing is adopted along two margins. First, people with higher values of going out, $y$, trade-off testing against going out without a test. Testing is altruistic, in that these people would otherwise have gone out, but now self-isolate to avoid infecting others. Second, people with lower values of $y$ trade-off testing against self-isolation. A negative test result gives them the required assurance that they are not contagious. The cheaper tests are the more people switch to testing, along both margins. Moreover, the more accurate the test (lower $\alpha$ and/or $\beta$ ), the more people switch to testing, too.

Overall, testing adds an option to individuals and therefore increases the utility of those who adopt it. The question remains what effect testing has on the equilibrium infection risk. In the online appendix we show that the CO-curve becomes

$$
\mu_{C O}(\phi)=\frac{\frac{\phi}{1+2 \phi}\left[1-\Omega\left(\widehat{Y}_{2}\right)\right]+\frac{\phi \beta}{1+2 \phi+\bar{\phi} \alpha}\left[\Omega\left(\widehat{Y}_{2}\right)-\Omega\left(\widehat{Y}_{1}\right)\right]}{\frac{1+\phi}{1+2 \phi}\left[1-\Omega\left(\widehat{Y}_{2}\right)\right]+\frac{1+\phi \beta}{1+2 \phi+\bar{\phi} \alpha}\left[\Omega\left(\widehat{Y}_{2}\right)-\Omega\left(\widehat{Y}_{1}\right)\right]}
$$

An important intuition (and the reason why this function looks somewhat complex) is that in addition to the $G O$ types, the pool of people going out in public now includes $T E$ types. Many of them tested negative correctly, and they help to reduce the average contagiousness of the public pool. Note that they only enter in the denominator of the above expression. As for those who had a false negative test, they enter both numerator and denominator, and increase the average contagiousness of the public pool.

Proposition 4 Higher values of $c$, $\alpha$, and $\beta$ shift the CO-curve outwards. The equilibrium infection risk $\phi^{*}$ is thus increasing in $c, \alpha$, and $\beta$.

Voluntary testing by private individuals has an important effect on the equilibrium infection risk. The more accurate and the cheaper tests are, the more people use them. This means that fewer of the people who go out are contagious, and thus fewer infections occur. This is true even if tests are imperfect, i.e., if some of the people going out do so on the basis of a false negative.

Unlike the typical SIR/SEIR models, our model is based on utility maximization. Consequently, we can analyze social welfare by looking at the sum of utilities across all agents in the economy 9 Specifically, we define the social welfare function as follows:

$$
W=\int_{0}^{\widehat{Y}_{1}} U^{S I} d \Omega(y)+\int_{\widehat{Y}_{1}}^{\widehat{Y}_{2}} U^{T E} d \Omega(y)+\int_{\widehat{Y}_{2}}^{\infty} U^{G O} d \Omega(y) .
$$

Proposition 5 The social welfare function is decreasing in $c, \alpha$, and $\beta$.

Proposition 5 is a central finding of this paper. It says that social welfare is increasing as the price of tests comes down. Those individuals buying the test clearly prefer lower prices. Moreover, as shown

\footnotetext{
${ }^{9}$ This is a standard economic approach for measuring social welfare. It has the benefit of being simple and transparent, but it misses the deeper debates about inequality.
} 


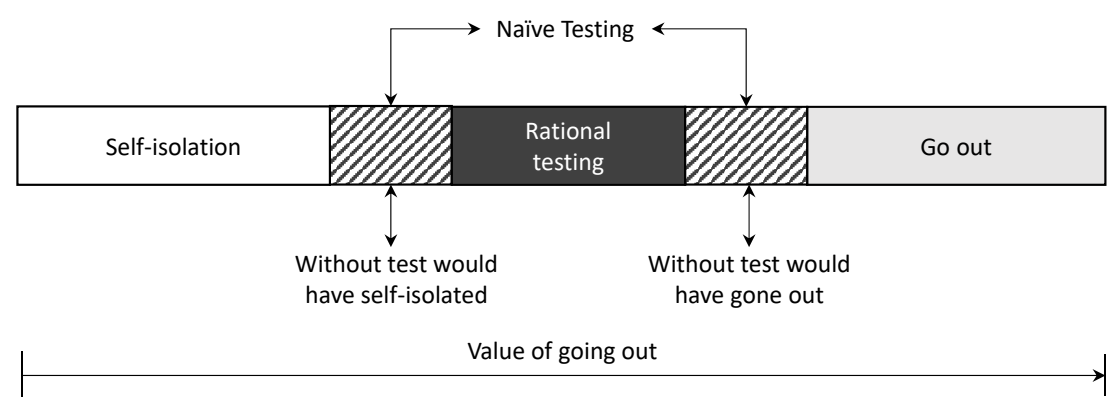

Figure 4: Comparison of Equilibrium Choices with Naïve Customers

in Proposition 4, the risk of infection comes down with lower test prices, which benefits everyone else too. Maybe the most remarkable part of Proposition 5 is that this simple logic holds true irrespective of testing inaccuracies, i.e., it holds for all values of $\alpha$ and $\beta$. Obviously, higher accuracy is still welcome. Indeed, Proposition 5 finds that social welfare always increases when tests become more accurate (i.e., lower $\alpha$ and $\beta$ ).

\section{Extensions}

\subsection{Naïve Customers}

A natural concern is that all individuals are assumed to fully understand the accuracy of the test. One of the concerns in the medical mainstream is that inaccurate tests can mislead the public. This argument boils down to people not understanding the true accuracy of test results. Thus, we consider a simple model extension where some fraction $\xi$ of the population is naïve and believes tests to be perfect. Such naïve people do not understand type I/II errors and thus put too much faith into test results. We ask how this affects individual choices and the equilibrium infection risk.

Figure 4 shows the difference between naïve and rational individuals. The key insight is that two types of naïve people buy the test, even though they wouldn't have done so if they fully understood its accuracy: ( $i$ ) those who would have self-isolated (lower $y$, on the left side in Figure 4), and (ii) those who would have gone out (higher $y$, on the right side in Figure 4). The first types are precisely those that the medical community typically worries about, where a false negative test encourages them to go out when they shouldn't. However, our analysis shows that there is also a second type that would otherwise have gone out. Their naïveté makes them buy the test, even though they selfishly wouldn't have done so if they fully understood its accuracy. Whenever they get a positive test result (correct or false), they self-isolate, something that everyone would welcome.

Ceteris paribus it is always better to be rational than naïve from an individual utility perspective. The interesting question is how the presence of naïve people affects the equilibrium infection risk. 
Proposition 6 A higher fraction of naïve individuals (higher $\xi$ ) shifts the CO-curve backward. The equilibrium infection risk is decreasing $\xi$.

This surprising result says that if more people are naïve, the equilibrium infection risk goes down. Naïve people overestimate the power of the test, and use it even though their rational alter-ego wouldn't. This increases the number of infected people self-isolating and decreases the average contagiousness of the people going out, thereby reducing the equilibrium infection risk.

\subsection{Required Tests}

Our model assumes that testing affects the decision to go out, but not the utility of doing so. In reality, testing may change what people do, and thus may also affect the utility of going out. For example, people may do certain things only once they had a negative test result, such as visiting a fragile relative. Access to certain events (e.g., music concerts) might also require having a negative test result. Suppose thus that people going out get $y$ with a negative test, but without it they only get $(1-\tau) y$. A higher $\tau$ represents greater limitations to people without tests.

Proposition 7 More individuals choose testing when $\tau$ increases. This reduces the equilibrium infection risk. Formally, $\widehat{Y}_{2}(\tau)$ is increasing and $\phi^{*}(\tau)$ decreasing in $\tau$.

Facing more limitations convinces more people to test. This stops more infected people from going out, leading to a lower equilibrium infection risk.

\subsection{Partial Isolation}

So far we considered three possible strategies for individuals: (i) go out indiscriminately $(G O)$, (ii) use test and only go out if test is negative (TE), and (iii) always self-isolate $(S I)$. In this section we consider an additional strategy that we call the partial isolation $(P I)$ strategy. This combines elements of the $G O$ and $S I$ strategies. Specifically, after going out at some date $t$, an individual stays at home at $t+1$ to see whether $\mathrm{s} / \mathrm{he}$ is infected. If $\mathrm{s} / \mathrm{he}$ is still heathy at $t+2, \mathrm{~s} / \mathrm{he}$ is certain not to be contagious, and goes out again without fear of remorse. The expected utility is given by

$$
U_{\phi}^{P I}=\phi \delta\left[-z+\delta\left(y+\delta U_{\phi}^{P I}\right)\right]+\bar{\phi} \delta\left(y+\delta U_{\phi}^{P I}\right)
$$

which in steady state implies

$$
U_{\phi}^{P I}=\frac{\delta[(1-\phi \bar{\delta}) y-\phi z]}{1-(1-\phi \bar{\delta}) \delta^{2}} .
$$

Proposition 8 There exists a threshold $z^{\prime}$, with $z^{\prime}<z^{*}$, such that partial isolation $(P I)$ is chosen by some individuals when $z \leq z^{\prime}$. The optimal choices are then as follows: 
(i) For all $y<\widehat{Y}_{1}^{\prime}$ individuals completely self-isolate $(S I)$.

(ii) For all $\widehat{Y}_{1}^{\prime} \leq y<\widehat{Y}_{1}^{\prime \prime}$ individuals partially self-isolate $(P I)$.

(iii) For all $\widehat{Y}_{1}^{\prime \prime} \leq y<\widehat{Y}_{2}$ individuals test (TE).

(iv) For all $y \geq \widehat{Y}_{2}$ individuals go out $(G O)$.

The critical value $\widehat{Y}_{1}^{\prime}$ does not depend on $c, \alpha$, and $\beta$, while $\widehat{Y}_{1}^{\prime \prime}$ is increasing in $c$, $\alpha$, and $\beta$.

Proposition 8 shows that partial isolation would only be an equilibrium choice for some individuals if the illness is sufficiently mild (low $z$ ). Technically the model becomes much more cumbersome because the public pool (and thus the contagiousness curve) involves additional types. Still, in the online appendix we derive a mild sufficient condition that ensures that our key insights from Proposition 4 continue to hold.

\subsection{Endogenous Encounters and Shielding}

To derive the infection curve we assumed a fixed number of encounters $M$, and an exogenous shielding technology $\lambda$. In reality we might expect these to depend on the infection risk. For instance, people will try to reduce the number of meetings they take when infection rates go up, or they may avoid some of the most crowded places. Similarly, people might shield themselves more if infection rates rise, such as wearing masks and keeping safe distance. In the online appendix we briefly show that allowing $M$ or $\lambda$ to be endogenous does not change the basic insights from the model. The main effect of making them endogenous is that the IN curve becomes steeper. This reduces the equilibrium infection risk, but has no other effect on the model.

\section{Discussion}

In this section we discuss the interpretation of our results, address robustness, and explore possible avenues for future research. The main objective of this short paper is to provide a theoretical framework for assessing the economic role of voluntary self-testing in the context of an on-going pandemic. The types of tests considered here are very different from the diagnostic tests used by trained staff in standard clinical setting. Our tests are meant to be self-administered at home by private individuals.

Our theory generates three broad insights. First, the model uncovers two margins along which private individuals opt into testing. There are those people who, in the absence of testing, would self-isolate, and those who would always go out. Second, the price and ease of use of a test plays an important role for the equilibrium infection risk. A positive test result convinces high-risk people to self-isolate. Moreover, a negative test result enables low-risk people to go out, which reduces the average contagiousness in public places. Third, the model sheds light on the role of diagnostic accuracy, showing that imperfect tests still 
help to reduce the equilibrium infection risk. One of the more surprising results is that the presence of naïve people actually reduces the equilibrium infection risk.

We obtain our key results in an analytically tractable steady-state model. This requires several sets of limiting assumptions. To begin with, our steady-state model cannot capture the dynamics of a pandemic over time, such as its peaks and troughs. However, our focus is on what the "new normal" economy might look like, if and when pandemics become an on-going economic threat.

We use strong simplifying assumptions about the medical progression of the disease. For example, in our model no one ever dies. Adding death to the model would negate any steady state analysis, because the density of people in the various regions of Figure 3 would change over time. However, as long as the death rate remains low, these density changes are slow moving, and have minimal impact on the equilibrium. Other limiting assumptions include the absence of any immunity, and the fixed duration of each stage of disease progression. If the purpose of the model were a realistic description of a pandemic, then these limitations would be more serious. However, our focus here is to understand the economic decisions of private individuals, and how they get aggregated in equilibrium. Our simplifying medical assumptions thus allow us to focus on these economic mechanisms.

This brings us to the most important set of simplifying assumptions, about economic behaviors. Let us discuss three such simplifications, and how future research might tackle them. First, for analytical tractability we only model one dimension of heterogeneity, namely the utility of going out $(y)$. This is an important dimension because different people have different opportunity costs of self-isolating (e.g., some people can easily work from home, others risk losing their economic livelihood). However, there could also be heterogeneity in other model dimensions: different people could have different levels of remorse $(x)$, fragility $(z)$, or difficulties self-administering a test $(c)$. People may also have different beliefs about the prevalence of the disease, the risk of infection, and so on. Future research could enrich the model along several of these dimensions.

Second, in our model there is only one way of getting tested, namely self-testing at home. In reality there is also testing in public places (e.g., transportation hubs), testing in the workplace, and obviously testing in clinical settings. Moreover, we are currently witnessing the development of novel testing methods, such as group testing (e.g., collective saliva collections) and local environment testing (e.g., surfaces, sewage). Future research might examine the relative advantages of these alternative approaches, and how they all interact with each other.

Third, our analysis focuses on the determinants of the equilibrium infection risk. A promising line for future research would be to perform a more comprehensive policy analysis. Our results suggest the possibility of government subsidies for voluntary self-testing. A more detailed policy analysis could look at the entire social cost and benefit analysis. Clearly there are fiscal costs to government subsidies, but there may also be additional considerations. Should all people get equal access to subsidized testing, or should certain groups be favoured? Should subsidies go to the more fragile (e.g., older people), or to the more outgoing (e.g., younger people)? In conclusion, we note that we are only beginning to understand the economic structures and choices of a new normal economy with ongoing pandemics. 


\section{References}

Agrawal, A., J. Gans, A. Goldfarb, and M. Lederman, 2020. The CEO's Guide to Safely Reopening the Workplace. MIT Technology Review, May 28th.

Berger, D., K. Herkenhoff, and S. Mongey, 2020. An SEIR Infectious Disease Model with Testing and Conditional Quarantine. Mimeo, Duke University.

Brauer, F., and C. Castillo-Chavez, 2012. Mathematical Models in Population Biology and Epidemiology, vol. 2 of Texts in Applied Mathematics. Springer.

Eichenbaum, M. S., S. Rebelo, M. Trabandt, 2020. The Macroeconomics of Testing and Quarantining. NBER Working Paper No. 27104.

Farboodi, M., G. Jarosch, and R. Shimer, 2020. Internal and External Effects of Social Distancing in a Pandemic. NBER Working Paper No. 27059.

Gans, J. S., 2020a. Economics in the Age of COVID-19. MIT Press.

Gans, J. S., 2020b. The Economic Consequences of $\hat{R}=1$ : Towards a Workable Behavioural Epidemiological Model of Pandemics. NBER Working Paper 27632.

Gersovitz, M., and Hammer, J. S., 2004. The Economical Control of Infectious Diseases. The Economic Journal 114(492), 1-27.

Hellmann, T., 2020. CDL Recovery - Some Lessons Learned. Mimeo, University of Oxford.

Meyer, R., and A. Madrigal, 2020. How to Test Every American for COVID-19, Every Day - The plan that could give us our lives back. The Atlantic, August 14th 2020.

Peto J. et al., 2020. Weekly COVID-19 testing with household quarantine and contact tracing is feasible and would probably end the epidemic. Royal Society Open Science, 7: 200915.

Plater, R., 2020. As Many as 80 Percent of People with COVID-19 Aren't Aware They Have the Virus. Healthline, https://www.healthline.com/health-news/50-percent-of-people-1 with-covid19-not-aware-have-virus

Rachel, L., 2020. An Analytical Model of Covid-19 Lockdowns: Equilibrium Mitigation Flattens the Epidemic Curve, Optimal Lockdown Does Not. Mimeo, London School of Economics and Political Science.

Toxvaerd, F., 2020. Equilibrium Social Distancing. Cambridge-INET Working Paper Series No: 2020/08. 